\title{
Duration measurement of vocants in infants with Cochlear Implants
}

\author{
Paris Binos ${ }^{1}$, Chryssoula Thodi ${ }^{2}$, George Psillas ${ }^{3}$, Paris Vogazianos ${ }^{2}$ \\ ${ }^{1}$ Department of Rehabilitation Sciences, Cyprus University of Technology, Cyprus \\ 2Department of Health Sciences, European University Cyprus, Cyprus \\ ${ }^{3}$ Department of Medicine, Aristotle University of Thessaloniki, Greece \\ https://doi.org/10.36505/ExLing-2020/11/0014/000429
}

\begin{abstract}
Vocants are precursors to speech and are facially neutral. The presence of these speechlike vocalizations was evident during the precursors to mature phonology called "protophones". The prosodic feature of duration of the nuclei plays a crucial role in the shift of prelexical to mature speech, since speech intelligibility is closely related to the control of duration. The aim of this work is to determine whether cochlear implants (CIs) positively trigger language acquisition and the development of verbal skills. Recent literature findings are compared and discussed with the performance of two Greek congenitally hearing-impaired infants who were matched with three normal-hearing $(\mathrm{NH})$ infants. This work highlighted an important weakness of the prosodic abilities of young infants with CIs.
\end{abstract}

Keywords: vocants, prosody, duration, deaf, cochlear implant

\section{Introduction}

Language and communication are primarily based on hearing. Hearing deprivation interferes with acquisition of crucial acoustic cues, limits word comprehension, and lowers oral language performance it . Although paediatric cochlear implantation provides measurable benefits on language perception, persistent high heterogeneity of findings prevents a thorough understanding of the process (Spencer, Marschark, Spencer, 2011).

Vowel duration during babbling is crucially important for future speech and language development: shorter vocal duration is related to mature adult-like vocalizations (Kent, Forner, 1980). There is an extensive work related to midfront, low-front and central vowels of children with normal hearing $(\mathrm{NH})$ (Fernald 2004, Katz, Cohn, Moore, 1996, Kitamura, Lam, 2009) during the babbling stage but there are only few reported studies focused on vocant prosodic features (quasivowels and full vowels) of infants with severe-toprofound hearing loss despite their clinical importance (Binos, Thodi, Vogazianos, Psillas, Constantinidis, 2020).

ExLing 2020: Proceedings of 11 th International Conference of Experimental Linguistics, 12-14 October 2020, Athens, Greece 


\section{Aim of the study}

The aim of this literature overview was to present recent findings on suprasegmental duration features in young CI recipients based on very early speech transitions called vocants, and to compare a recent study based on Greek language (Binos et al. 2020) other studies exploring similar phenomena.

\section{Method}

We reviewed published peer-reviewed studies from the last ten years identified via a PubMed/Medline search. The keyword combination "vowel duration" revealed seventeen papers and "vowel duration cochlear implants" revealed two papers. . Inclusion criteria included whether the papers were research reports, clinical trials, meta-analysis, randomized controlled trials, reviews and systematic reviews and only if a full-text access was possible; the papersshould report on infants with CI with speech analysis in the babbling stage. This focus on studies from the last ten years was dictated by recent technological developments that shaped cochlear implants capabilities.

Based on our inclusion criteria studies related to adults or normal-hearing participants were excluded. The papers included (Neumeyer, Harrington, Draxler, 2010, VanDam, Ide-Helvie, Moeller, 2011).

The Greek language study (Binos et al, 2020). Binos et al., (2020), analyzed vocant productions based on spontaneous vocalizations using a combination of acoustical and auditory characteristics. Reflexive or vegetative sounds were excluded.Our CI participants had deafness of unknown etiology and no other disabilities and were enrolled in auditory-oral programs.

Prior to implantation, the infants of the Greek study had an average unaided hearing loss of 95-110 dBHL, while the post implantation pure-tone audiometry (PTA) was 10-38 dBHL. A wideband spectrographic analysis was performed selecting segments from voice onset to the loss of audible signal and the decrease of higher formant energy. To match children for hearing experience, infants with CIs were chronologically older and more developmentally mature than $\mathrm{NH}$ peers. Due to the high $\mathrm{F}_{0}$ of infants' speech, a large analysis bandwidth $(450 \mathrm{~Hz})$ was used for the spectrographic analysis. The vocant onset and offset were measured via wideband spectrographic analysis from the voicing onset (first glottal pulse) to the loss of audible signal and the decrease in the energy of higher formants (F2). The decrease of energy was visualized through wideband spectrograms where a lowering of F2 was visible while the oral cavity was enlarged because of tongue retraction (Binos et al, 2020).

\section{Results}

During the Binos et al (2020) analysis the Kolmogorov-Smirnov test was used for sample sizes greater than 50 and the Shapiro-Wilk test for samples smaller 
than 50 to check for data distribution. The data analyzed did not follow the normal distribution for the duration variable. The Levene Test of Equality of Variance was carried out for the duration variable of the groups and the results showed no homogeneity of variances for the duration variable making the Median test more appropriate than the Mann Whitney test. The results $(\delta=$ 512.5, $\left.\chi^{2}=29.020, \mathrm{p}<.001\right)$ displayed a major weakness for the CI participants on the duration performance. The average vocant duration for $\mathrm{NH}$ infants was significantly lower from the $\mathrm{CI}$ infants.

\section{Discussion}

The studies of VanDam, Ide-Helvie and Moeller (2011) and Yang, Brown, Fox et al (2015) agree with the Greek study since the lower the chronological age the longer vocal duration. The VanDam et al (2011) study included groups of CI users ages8-9 years old and the duration of vowels was found longer but only for typical development children when the vowels were followed by consonants (type CVC). In the Yang et al (2015) study pre-lingually deafened CI children (2.9-8.3 years old) produced seven Mandarin vowels longer than the $\mathrm{NH}$ children did. Longer duration measured for the isolated vowels $/ \mathrm{e}, \alpha /, / \mathrm{i}$ $\alpha /$ of CI users in German (Neumeyer et al, 2010). The Greek study broadens these findings to younger $\mathrm{CI}$ individuals.

Longer vocant duration in young CI participants is considered a poor predictor for speech production skill development. Recent studies associate shorter duration of vowels with greater speech maturity and greater intelligibility. The clinical implications of our finding are critical for articulatory positions and acoustic outputs, as habilitation models for young CIs should be adjusted accordingly.

\section{Acknowledgements}

The authors warmly thank all families that participated in the Greek study. We also thank the University Hospital of AHEPA in Thessaloniki, Greece. Our special thanks go to the ENT Clinic for their continuous support and participation.

\section{References}

Binos, P., Thodi, C., Vogazianos, P., Psillas, G., Constantinidis, J. 2020. An acoustic and auditory analysis of vocants in infants with cochlear implants. Logopedics Phoniatrics Vocology, DOI: 10.1080/14015439.2020.1724325.

Fernald, A. 2004. Hearing, listening, and understanding auditory development in infancy. In Blackwell handbook of infant development. London: Blackwell Publishing: 3570.

Neumeyer, V., Harrington, J., Draxler, C. 2010. An acoustic analysis ofthe vowel space in young and old cochlear-implant speakers. Clin Linguist Phon 24(9), 734-741. 
Katz, G., Cohn, J., Moore, C. 1996. A combination of vocal f0 dynamic and summary features discriminates be- tween three pragmatic categories of infant-directed speech. Child Dev 67, 205-217.

Kent, R., Forner, L. 1980. Speech segment durations in sentence recitations by children and adults. J Phon 8(2), 157-168.

Kitamura, C., Lam, C. 2009. Age-specific preferences for infant-directed affective intent. Infancy 14(1), 77-100.

Spencer, P.E., Marschark, M., Spencer, L.J. 2011. Cochlear implants: Advances, issues and implications. In The Oxford Handbook of Deaf Studies, Language and Education. (eds.) (vol. 1, 2nd edn). New York, NY: Oxford University Press: 452-471.

Yang, J., Brown, E., Fox, R., et al. 2015. Acoustic properties of vowel production in prelingually deafened Mandarin-speaking children with cochlear implants. J Acoust Soc Am. 138(5), 2791-2799. 